\title{
A SPONTANEOUS INTRAMURAL SMALL BOWEL HAEMATOMA
}

\author{
Sandamalee N K, Ranasinghe D D, Epa W A \\ Department of Radiology, Colombo South Teaching Hospital
}

\begin{abstract}
Spontaneous haemorrhage into small bowel wall leading to obstruction is a rare acute presentation among haemophilic patients. We present a case of a 46 year old male with haemophilia $\mathrm{B}$, admitted to the surgical casualty ward with an acute abdominal pain suspicious of small bowel obstruction which was confirmed on erect abdominal radiograph and abdominal sonography. Contrast enhanced CT [CECT] abdomen revealed a segment of circumferentially thickened small bowel wall suggestive of bowel wall heamatoma. However, mild but appreciable contrast enhancement of the affected bowel wall raised the possibilities of a neoplastic or inflammatory process as differentials. Subsequent surgical intervention with resection of bowel after correction of factor IX and histology revealed intramural heamatoma of small bowel with no evidence of an inflammatory or neoplastic process. We believe that the bowel wall enhancement in CECT could be attributed to the enhancement of intact mucosa and sub mucosa predominating over non enhancing heamatoma which can be considered a diagnostic pitfall.
\end{abstract}

Keywords: Small bowel, Intramural, Heamatoma, Haemophilia, Spontaneous

Correspondence: N. Kusala Sandamalee. <Kusala_5@yahoo.com >

Copyright: Sandamalee N K 


\section{Case report $\mid$ SLJR}

\section{Introduction}

Non traumatic spontaneous small bowel heamatoma is a rare occurrence ${ }^{1,4}$. It is more common in patients receiving anticoagulation or with bleeding disorders ${ }^{1,3}$. Computed tomography (CT) is the imaging technique of choice which shows mural hyper-density in a circumferentially thickened small bowel segment ${ }^{1,3,4}$. Early recognition of the condition may prevent unnecessary surgery ${ }^{1,3,5}$.

\section{Case Report}

A 46 year old male presented to the casualty surgical ward with abdominal pain and vomiting for 5 days duration. He had no bowel motions for 3 days but flatus was passed. There was no history of fever. He was a diagnosed patient of Haemophilia B. There was no past history of surgery. Initial laboratory investigations revealed WBC count of $20 \times 103 / \mu 1$ with $85.9 \%$ neutrophils, $12.3 \mathrm{~g} / \mathrm{dl}$ haemoglobin, $467 \times 103 / \mu 1$ platelet count and 1.03 INR. Serum electrolyte counts were normal with $\mathrm{K}+3.7 \mathrm{mmmol} / \mathrm{L}$ (ref range 3.5-5.1) and $\mathrm{Na}+140 \mathrm{mmol} / \mathrm{L}$ (ref range 136-145). Urgent erect $X$ ray abdomen showed multiple air fluid levels $(>3$, each $>3 \mathrm{~cm}$ ) suggesting intestinal obstruction (Figure 1) and the ultrasound scan (USS) of the abdomen showed a long segment of small bowel with absent peristalsis and thickened wall. Abrupt transition was seen from normal bowel to thickened bowel. Bowel wall vascularity was not seen on Doppler USS performed using the high frequency linear transducer (Figure 2). Emergency laparotomy was contemplated on the provisional diagnosis of bowel gangrene but had to be postponed until factor IX was available.

However, subsequent sonography with low frequency curvilinear transducer and

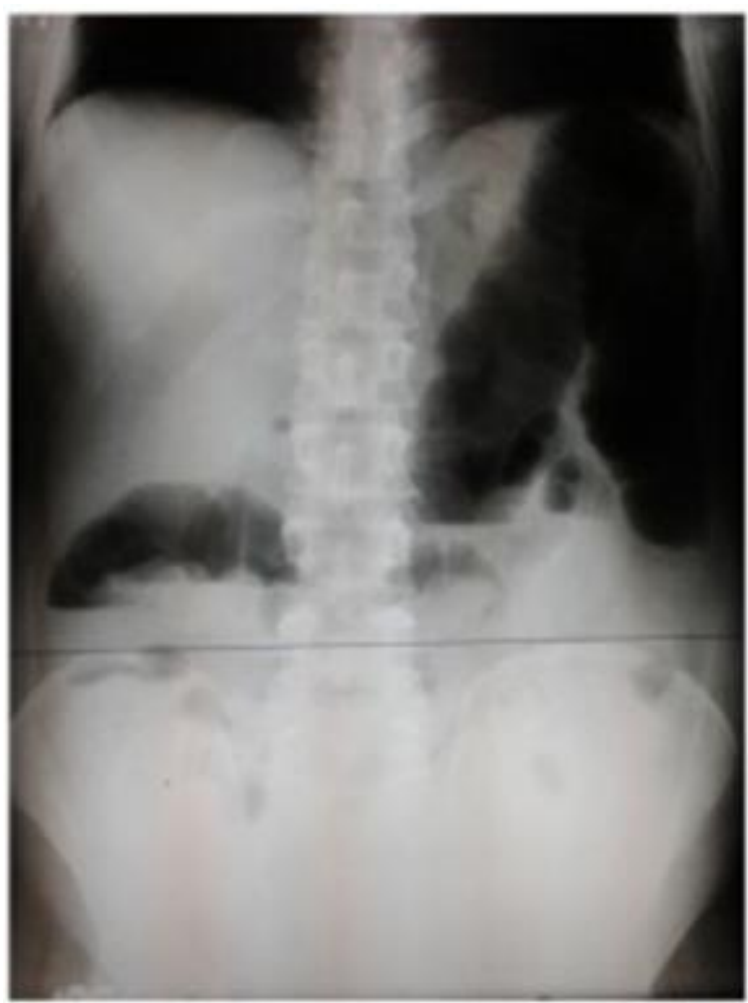

Figure 1: Erect AXR; dilated small bowel

with fluid levels

optimized color Doppler parameters showed bowel wall vascularity, thus surgery was delayed until factor IX deficiency was corrected. CECT abdomen was performed to assess the extent of involvement of the bowel and to exclude bowel gangrene. It revealed a long segment of distal jejunum and proximal ileum with thickened enhancing wall. Adjacent mesenteric hyperaemia was observed too. No local or regional lymph adenopathy but there was mild ascites. No intra mural gas was seen in the bowel and the other bowel loops appeared unremarkable (Figures 3 and 4). CT findings of contrast enhancement of the bowel wall and lack of gas in the bowel wall ruled out the possibility of small bowel gangrene. Although in a 


\section{Case report SLJR}

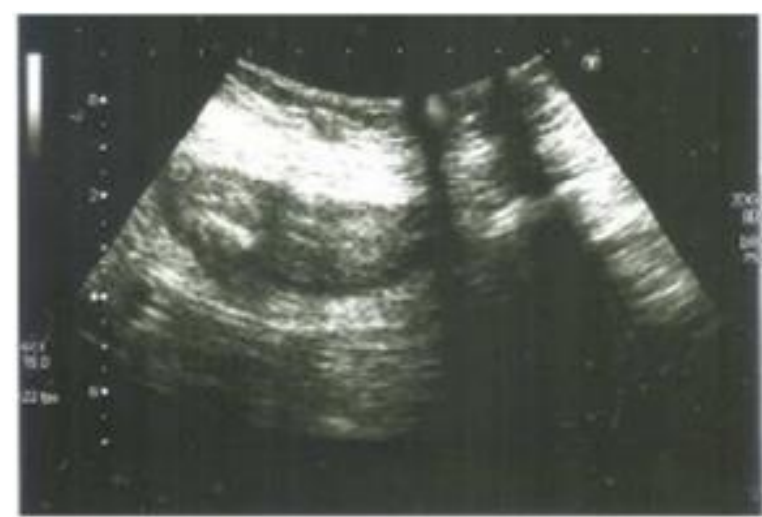

Figure 2: US scan; segment of small bowel with wall thickening

patient with a bleeding diathesis, the logical conclusion of small bowel wall thickening is hemorrhage into the bowel wall, presence of mild but appreciable contrast enhancement prompted us to look for an alternative cause since hematoma per se is not expected to show contrast enhancement. Differential diagnosis was given as either neoplastic lesion such as primary small bowel lymphoma or inflammatory bowel pathology involving a long segment of small bowel.

Surgery was performed after correction of factor IX. A long segment $(45 \mathrm{~cm})$ of hyperaemic non gangrenous bowel was

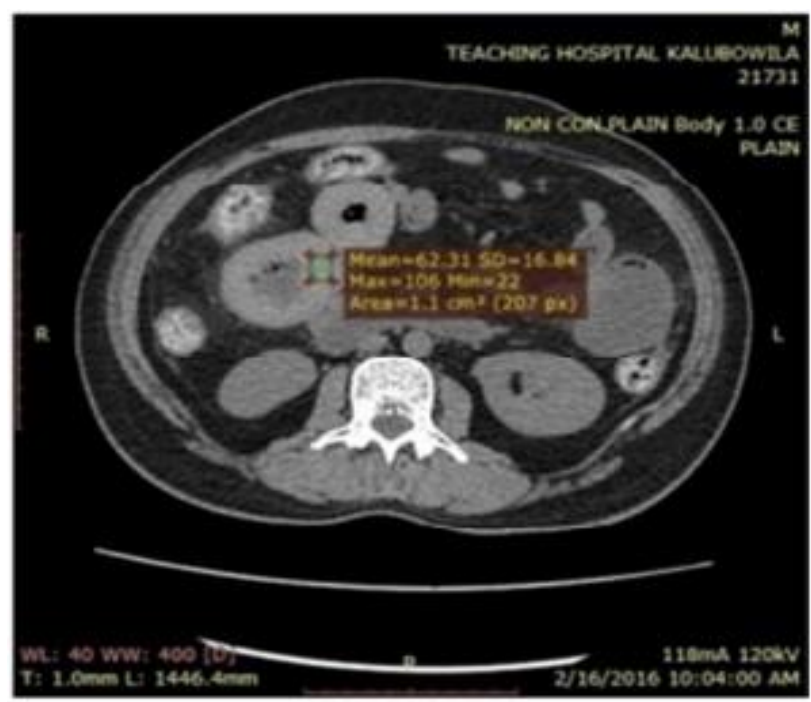

evident with intramural and mesenteric heamatomas (Figure 5). One and a half feet of thickened, condensed small was removed and end to end anastomosis was performed. Histology revealed intact viable mucosa and extensive haemorrhage in the lamina propria and submucosa with small haemorrhages in the mesentery (Figures 6,7,8).

\section{Discussion}

Spontaneous intramural small bowel heamatoma is a rare occurrence mainly found in over anti-coagulated patients or in patients with bleeding disorders ${ }^{1,3}$ similar to this case. It is most commonly seen in jejunum followed by ileum and duodenum and is only rarely seen in colon ${ }^{1,4}$. A single lesion is seen more commonly than multiple lesions ${ }^{1}$. Sonographic appearance of acute bowel wall heamatoma is described as thickened echogenic submucosa $\mathrm{a}^{1}$. However, this is a nonspecific finding which can be found in other conditions such as inflammatory bowel disease. The CECT findings are reported as homogenous symmetrical intramural thickening. Our patient had circumferentially thickened bowel wall on CT in concurrence

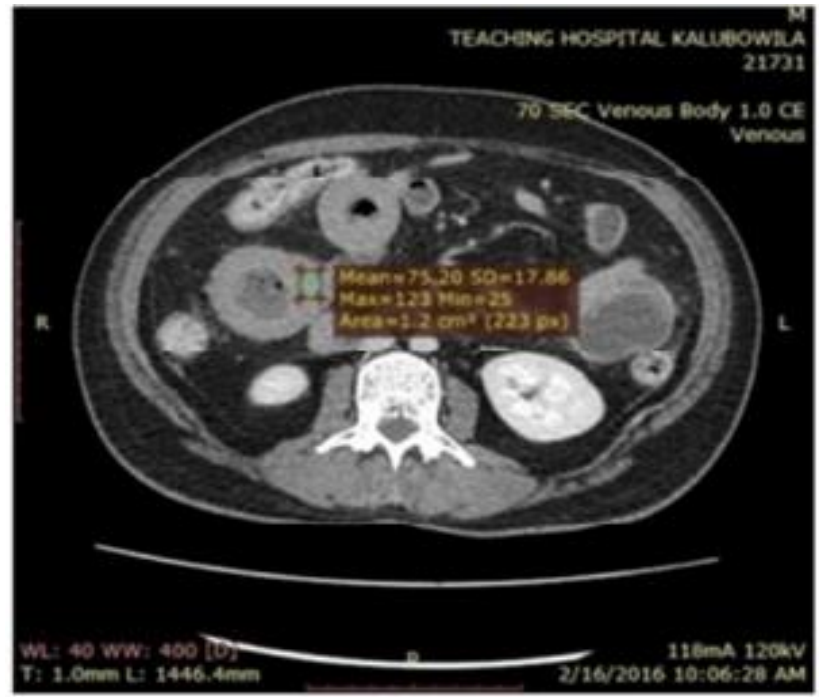

Figure 3: Pre and post contrast axial CT images - segment of small bowel with thickened wall 


\section{Case report $\mid$ SLJR}
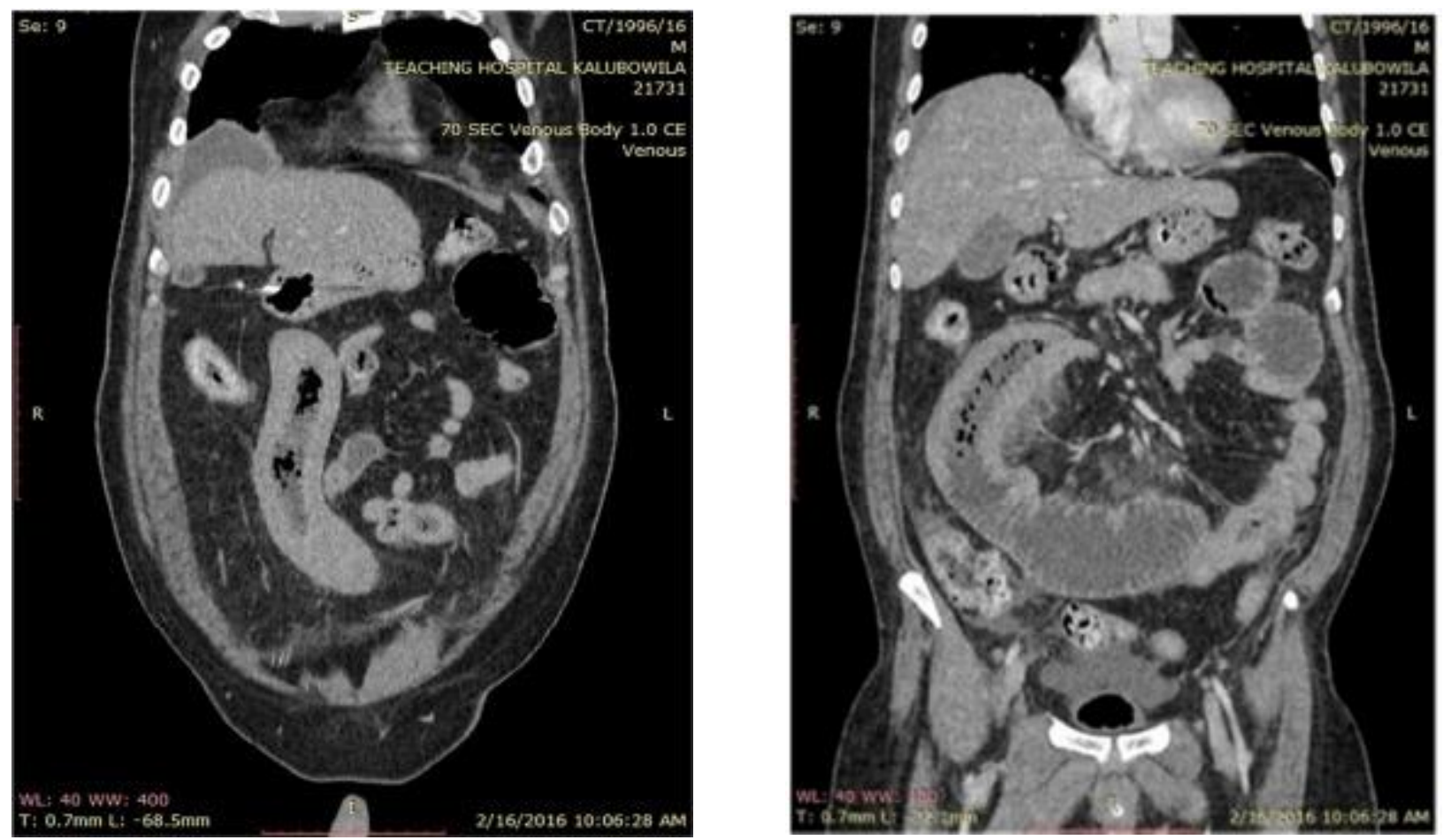

Figure 4: Coronal CT; segment of small bowel with thickened wall and mesenteric hyperaemia.

with literature. The thickening is further described as non-enhancing hyper-dense material within bowel wall in early stages. The hyper-density is reported to reduce after about 10 days when the heamatoma ages and an intramural cystic lesion becomes evident ${ }^{1}$. Reduction in attenuation is seen in centripetal fashion as the clot lyses ${ }^{2}$. The characteristic ring sign with a crescent of hyper attenuation may be seen on $\mathrm{CT}^{2}$. Inflammatory lesions of the small bowel also can lead to bowel wall thickening with enhancement. Therefore, without clinical history, the CT findings are nonspecific for differentiating from inflammatory causes of bowel wall thickening ${ }^{2}$. Although literature describes non enhancing mural thickening in cases of haemorrhage, in our patient there was enhancement of the thickened segment of bowel. In this case, the enhancement of the bowel walls on CECT deviated the diagnosis from bowel wall heamatoma towards inflammatory or neoplastic lesion since heamatoma per se is not expected to show contrast enhancement. Absence of significant drop in $\mathrm{Hb}$ level and the neutrophil leukocytosis too supported an inflammatory cause over haemorrhage. Patients with uncomplicated small segment of intramural bowel heamatoma can be treated medically avoiding unnecessary surgery ${ }^{3,5}$. The average length of involved bowel segment which could be managed successfully with medical treatment is given as less than $23 \mathrm{~cm}$ in the literature ${ }^{1,5}$. The involved segment in this patient was $45 \mathrm{~cm}$, therefore the index patient is probably a candidate for surgery. Postsurgical period was found to be uneventful.

\section{Take home point}

We believe that the mild contrast enhancement seen in the bowel wall during CECT was a result of enhancement of intact viable bowel mucosa predominating over un enhanced heamatoma mimicking a neoplastic 


\section{Case report $\mid$ SLJR}

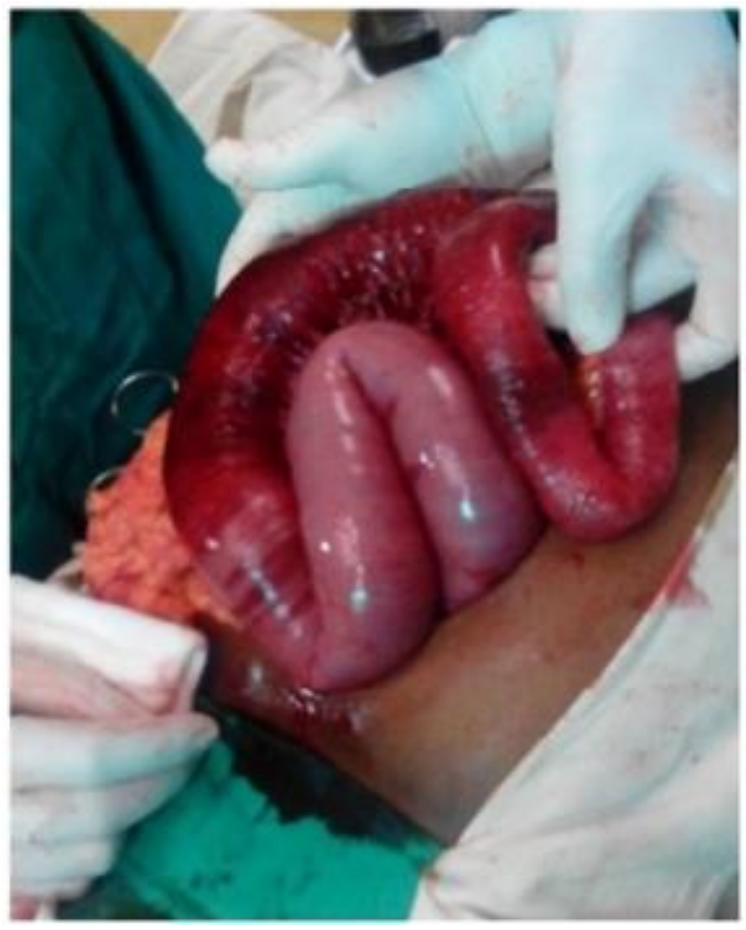

Figure 5: Segment of hyperaemic small

bowel during surgery

or inflammatory process and should be considered as a pit fall, to be avoided when interpreting CECT images of suspected intra mural haemorrhage of bowel.

\section{Acknowledgement}

We would like to thank the patient for consenting for publication, Dr. Imitiaz Ismail; Consultant Surgeon and Dr. Priyangani Amarabandu; Consultant Histopathologist.

\section{References:}

1. Maher A. Abbas, Joseph M. Collins and Kevin W. Olden.Spontaneous Intramural Small-Bowel Hematoma: Imaging Findings and Outcome. American Journal of Roentgenology 2002: 179:1389-94.

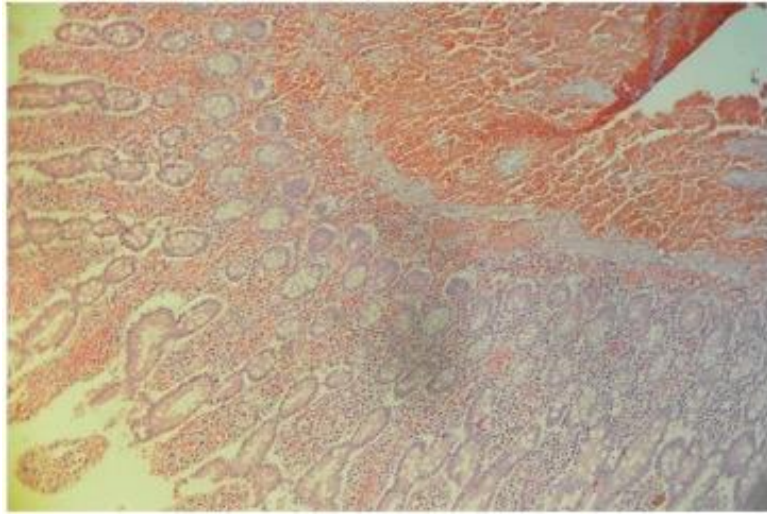

Figure 6: Microscopy; haematoma within submucosa.

2. Mourad Boudiaf, Philippe Soyer, CarineTerem, Jean Pierre Pelage, Emmanuelle Maissiat and Roland RymerCT Evaluation of Small Bowel Obstruction Radiographics 2001;21:613624 RSNA.

3. Sinan Çarkman, Volkan Özben, Kaya Saribeyoğlu, Erkan Somuncu, Sabri Ergüney, Uğur Korman, Salih Pekmezci Spontaneous intramural hematoma of the small intestine Turkish journal of trauma \& emergency surgery: TJTES (Impact Factor: 0.38). 03/2010; 16(2):165-9.

4. R Dibbad, M Deshmukh, H Merchant, S ShikhareSpontaneous Intramural Hematoma of the Small Bowel-A complication of Anticoagulant therapyISPUB.com / IJRA/12/1/6206.

5. Abbas MA, Collins JM, Olden KW, Kelly KA. Spontaneous intramural small-bowel hematoma: clinical presentation and longterm outcome. Arch Surg. 2002; 137:306- 10. 